\title{
Morphological Characterization of Cherry Rootstock Candidates Selected from Central and East Black Sea Regions in Turkey
}

\author{
Aysen Koc, ${ }^{1}$ Zumrut Celik, ${ }^{2}$ Mustafa Akbulut, ${ }^{3}$ Sukriye Bilgener, ${ }^{4}$ Sezai Ercisli, ${ }^{5}$ \\ Mehmet Gunes, ${ }^{6}$ Resul Gercekcioglu, ${ }^{6}$ and Ahmet Esitken ${ }^{7}$ \\ ${ }^{1}$ Department of Horticulture, Faculty of Agriculture and Natural Sciences, Bozok University, Yozgat, Turkey \\ ${ }^{2}$ Ministry of Food, Agriculture and Livestock, Ataturk Orman Ciftligi, Ankara, Turkey \\ ${ }^{3}$ Faculty of Agriculture and Natural Science, Recep Tayyip Erdogan University, Rize, Turkey \\ ${ }^{4}$ Department of Horticulture, Faculty of Agriculture, Ondokuz Mayis University, Samsun, Turkey \\ ${ }^{5}$ Department of Horticulture, Faculty of Agriculture, Ataturk University, Erzurum, Turkey \\ ${ }^{6}$ Department of Horticulture, Faculty of Agriculture, Gaziosmanpaşa University, Tokat, Turkey \\ ${ }^{7}$ Department of Horticulture, Faculty of Agriculture, Selcuk University, Konya, Turkey \\ Correspondence should be addressed to Sezai Ercisli; sercisli@gmail.com
}

Received 2 August 2013; Accepted 1 October 2013

Academic Editors: B. Chen and A. C. Manna

Copyright (C) 2013 Aysen Koc et al. This is an open access article distributed under the Creative Commons Attribution License, which permits unrestricted use, distribution, and reproduction in any medium, provided the original work is properly cited.

The use of rootstocks particularly for sweet cherry cultivars is of great importance for successful and sustainable production. Choosing the right cherry rootstocks is just as important as choosing the right cultivar. In this study, 110 sweet cherry, 30 sour cherry, and 41 mahaleb types displaying rootstock potential for sweet cherry cultivars were selected from Central and East Black Sea Regions in Turkey. The morphologic characteristics of the studied genotypes were compared with the standard clonal rootstocks PHL-A, MaxMa 14, Montmorency, Weiroot 158, Gisela 5, Gisela 6, and SL 64. A total of 42 morphological UPOV characteristics were evaluated in the selected genotypes and clonal rootstocks. The obtained data were analyzed by using principal component analysis and it revealed that eigenvalues of the first 3 components were able to represent $36.43 \%$ of total variance. The most significant positive correlations of the plant vigor were determined with leaf blade length and petiole thickness. According to the diversity analysis of coefficients, the $05 \mathrm{C} 002$ and $08 \mathrm{C} 039$ genotypes were identified as being similar (6.66), while the $05 \mathrm{C} 002$ and 55 S 012 genotypes were determined as the most distant genotypes (325.84) in terms of morphology.

\section{Introduction}

Turkey produces 438.550 tons of sweet cherries and 182.234 tons of sour cherries annually playing an important role in world sweet and sour cherry production. According to recent statistics, Turkey takes the first place in both sweet cherry production and export [1]. In Turkey, the mahaleb (Prunus mahaleb), wild sweet cherry (Prunus avium), and wild sour cherry (Prunus cerasus) seedlings are widely used as rootstocks for both sweet and sour cherry cultivars. Ercisli et al. [2] reported that $40 \%$ of the sweet cherry production in Turkey is carried out with wild sweet cherry seedlings, $30 \%$ with mahaleb seedlings, and 30\% with Gisela 5, Gisela
6, and SL 64 clone rootstocks. Sweet cherry scion cultivars have been selected over millennia for many reasons, while rootstocks have only recently received attention. Unlike scion cultivar development, evaluation of rootstock programs may require at least 10 years to be completed. However, improved new technologies have provided significant improvements in evaluation.

Genetic diversity (variation within species) is vital for the evolution of agricultural species and their adaptation to particular environments through a mixture of natural and human selection. In crop agriculture, for some species, this selection has led to the development of many thousands of "landraces" or "farmers" varieties. In addition to 
domesticated plants, wild species are important nutritionally and culturally to many people [3]. Germplasms collection and characterization are essential stages for breeding programs. Germplasms collection and characterization are generally performed by describing morphological characteristics. The international criteria of the International Union for the Protection of New Varieties of Plants (UPOV) and the International Plant Genetic Resources Institute (IPGRI) were created in order to remove unclear situation and to enable researchers to use common descriptive characteristics. The data from morphological traits were evaluated statistically by using principle component analysis (PCA), correlation, and morphological distance index.

Turkey is one of the most important genetic sources for cherries in the world and provides an important source of variation for plant breeding. However, as is the case with other species used in fruit production, our country does not have its own native cherry clonal rootstocks.

The aim of our study was to investigate genotypic variation among 110 sweet cherry, 30 sour cherry, and 41 mahaleb types selected among wild cherry populations in Central and East Black Sea Regions in Turkey that can potentially be used as rootstocks for cultivars in future.

\section{Materials and Methods}

With an initial extensive survey studies, a total of 459 wild accessions were collected from Central and East Black Sea Regions in Turkey and were preserved at the Black Sea Agricultural Research Institute located in Samsun province during 2006-2009. The survey studies were conducted at Amasya, Artvin, Giresun, Gümüşhane, Ordu, Rize, Samsun, Tokat, and Trabzon provinces of Black Sea region. Selected wild genotypes ( $P$. avium, $P$. cerasus, $P$. and mahaleb) were grafted by budding in the observation gardens. All types were identified using morphological characterization criteria of to UPOV (International Union for the Protection of New Varieties of Plants, Prunus Rootstocks 2002, TG/187/103.03.2007). Among 459 genotypes, we selected a total of 181 promising genotypes consist of 110 sweet cherry, 30 sour cherry, and 41 mahaleb and they were used for further analyses (Table 1). The morphologic characteristics of the studied genotypes were compared with PHL-A, MaxMa 14, Montmorency, Weiroot 158, Gisela 5, Gisela 6, and SL 64 worldwide reference clonal rootstocks. Morphological characteristics of the leaves were determined in July, while the morphological features of the shoots were determined in December. A total of 188 genotypes, including the selected genotypes and clonal rootstocks, were evaluated according to a total of 42 morphological and phenotypic characteristics (Table 2). Simple correlations, factor and cluster analyses, and scatter plots were prepared by using SPSS (version 20.0 for Windows). Factor analysis was performed by using the varimax factor rotating method. A dendrogram of the genetic similarities between the genotypes was compiled using the Ward method. The location data of selected genotypes was determined using GPS in the project area. These data were transferred in the GIS database and the distribution map was created using ArcGIS 9.2 software (Figure 1).
TABLE 1: The map data of types selected from Central and East Black Sea Regions in Turkey (C: sweet cherry, P. avium; S: sour cherry, $P$. cerasus; M: mahaleb, P. mahaleb; CA: C. angustifolia).

\begin{tabular}{|c|c|c|}
\hline Accession & Collection area (province-district) & $\begin{array}{c}\text { Altitude } \\
(\mathrm{m})\end{array}$ \\
\hline 05 C 002 & Amasya-Merkez & 1002 \\
\hline $05 \mathrm{C} 003$ & Amasya-Taşova & 640 \\
\hline 05 C 004 & Amasya-Taşova & 640 \\
\hline $05 \mathrm{C} 005$ & Amasya-Gümüşhacıköy & 821 \\
\hline 05 C 006 & Amasya-Gümüşhacıköy & 826 \\
\hline $05 \mathrm{C} 007$ & Amasya-Gümüşhacıköy & 875 \\
\hline 05 C 009 & Amasya-Gümüşhacıköy & 956 \\
\hline $05 \mathrm{M} 001$ & Amasya-Merkez & 449 \\
\hline 05 M 006 & Amasya-Merkez & 846 \\
\hline $05 \mathrm{M} 007$ & Amasya-Taşova & 1002 \\
\hline $05 \mathrm{M} 008$ & Amasya-Taşova & 767 \\
\hline 05 M 009 & Amasya-Taşova & 430 \\
\hline $05 \mathrm{M} 010$ & Amasya-Taşova & 430 \\
\hline $08 \mathrm{C} 001$ & Artvin-Yusufeli & 1554 \\
\hline 08 C 005 & Artvin-Yusufeli & 1409 \\
\hline $08 \mathrm{C} 007$ & Artvin-Yusufeli & 1445 \\
\hline 08 C 008 & Artvin-Yusufeli & 1411 \\
\hline $08 \mathrm{C} 017$ & Artvin-Şavşat & 1674 \\
\hline 08 C 018 & Artvin-Şavşat & 1974 \\
\hline $08 \mathrm{C} 022$ & Artvin-Şavşat & 1821 \\
\hline 08 C 028 & Artvin-Şavşat & 1622 \\
\hline 08 C 033 & Artvin-Borçka & 475 \\
\hline $08 \mathrm{C} 037$ & Artvin-Yusufeli & 1488 \\
\hline 08 C 039 & Artvin-Yusufeli & 1448 \\
\hline $08 \mathrm{C} 044$ & Artvin-Yusufeli & 1499 \\
\hline $08 \mathrm{C} 045$ & Artvin-Yusufeli & 1567 \\
\hline $08 \mathrm{C} 046$ & Artvin-Yusufeli & 884 \\
\hline $08 \mathrm{C} 053$ & Artvin-Merkez & 977 \\
\hline 08 C 056 & Artvin-Yusufeli & 1084 \\
\hline 08 C 057 & Artvin-Yusufeli & 1565 \\
\hline 08 S 002 & Artvin-Yusufeli & 1560 \\
\hline 08 S 003 & Artvin-Yusufeli & 675 \\
\hline $08 \mathrm{~S} 005$ & Artvin-Yusufeli & 1502 \\
\hline $28 \mathrm{C} 002$ & Giresun-Çanakçı & 427 \\
\hline $28 \mathrm{C} 005$ & Giresun-Tirebolu & 53 \\
\hline $28 \mathrm{C} 007$ & Giresun-Dereli & 1014 \\
\hline $28 \mathrm{C} 015$ & Giresun-Yağlidere & 256 \\
\hline $28 \mathrm{C} 016$ & Giresun-Yağlıdere & 68 \\
\hline $28 \mathrm{C} 020$ & Giresun-Bulancak & 717 \\
\hline $28 \mathrm{~S} 001$ & Giresun-Çanakçı & 417 \\
\hline $28 \mathrm{~S} 002$ & Giresun-Şebinkarahisar & 1247 \\
\hline $28 \mathrm{~S} 003$ & Giresun-Şebinkarahisar & 1121 \\
\hline $28 \mathrm{M} 001$ & Giresun-Şebinkarahisar & 1450 \\
\hline $28 \mathrm{M} 003$ & Giresun-Alucra & 1464 \\
\hline
\end{tabular}


TABle 1: Continued.

\begin{tabular}{|c|c|c|}
\hline Accession & Collection area (province-district) & $\begin{array}{c}\text { Altitude } \\
\text { (m) }\end{array}$ \\
\hline $28 \mathrm{M} 005$ & Giresun-Şebinkarahisar & 1270 \\
\hline 28 CA 001 & Giresun-Şebinkarahisar & 1270 \\
\hline $28 \mathrm{CA} 002$ & Giresun-Şebinkarahisar & 1217 \\
\hline $29 \mathrm{C} 001$ & Gümüşhane-Merkez & 1199 \\
\hline $29 \mathrm{C} 003$ & Gümüşhane-Torul & 1455 \\
\hline $29 \mathrm{C} 004$ & Gümüşhane-Kürtün & 1276 \\
\hline $29 \mathrm{C} 005$ & Gümüşhane-Kürtün & 1109 \\
\hline $29 \mathrm{C} 006$ & Gümüşhane-Torul & 1065 \\
\hline $29 \mathrm{~S} 001$ & Gümüşhane-Merkez & 1287 \\
\hline $29 \mathrm{~S} 003$ & Gümüşhane-Köse & 1654 \\
\hline 29 S 004 & Gümüşhane-Şiran & 1395 \\
\hline 29 S 005 & Gümüşhane-Torul & 1043 \\
\hline 29 M 001 & Gümüşhane-Merkez & 1281 \\
\hline $29 \mathrm{M} 004$ & Gümüşhane-Şiran & 1229 \\
\hline 29 M 006 & Gümüşhane-Torul & 1363 \\
\hline 29 CA 001 & Gümüşhane-Kelkit & 1464 \\
\hline 29 CA 002 & Gümüşhane-Kelkit & 1578 \\
\hline 29 CA 003 & Gümüşhane-Kelkit & 1710 \\
\hline 29 CA 004 & Gümüşhane-Şiran & 1329 \\
\hline $52 \mathrm{C} 004$ & Ordu-Aybastı & 771 \\
\hline $52 \mathrm{C} 005$ & Ordu-Kabataş & 623 \\
\hline 52 C 007 & Ordu-Mesudiye & 1203 \\
\hline $52 \mathrm{C} 008$ & Ordu-Gölköy & 919 \\
\hline $52 \mathrm{C} 009$ & Ordu-Kabadüz & 189 \\
\hline 52 C 011 & Ordu-Fatsa & 610 \\
\hline 52 C 013 & Ordu-Korgan & 1525 \\
\hline 52 C 014 & Ordu-Korgan & 1162 \\
\hline 52 C 015 & Ordu-Kumru & 579 \\
\hline 52 C 019 & Ordu-Kumru & 730 \\
\hline $52 \mathrm{C} 026$ & Ordu-Kabataş & 613 \\
\hline $52 \mathrm{C} 029$ & Ordu-Çamaş & 155 \\
\hline $52 \mathrm{C} 030$ & Ordu-Çamaş & 474 \\
\hline 52 C 031 & Ordu-Çatalpınar & 359 \\
\hline $52 \mathrm{C} 035$ & Ordu-Çatalpınar & 640 \\
\hline 52 C 038 & Ordu-Mesudiye & 1289 \\
\hline 52 C 039 & Ordu-Mesudiye & 1072 \\
\hline 52 C 042 & Ordu-Gölköy & 829 \\
\hline $52 \mathrm{C} 046$ & Ordu-Gürgentepe & 1015 \\
\hline $52 \mathrm{C} 050$ & Ordu-Gürgentepe & 1021 \\
\hline $52 \mathrm{C} 054$ & Ordu-Ulubey & 513 \\
\hline 52 C 056 & Ordu-Merkez & 219 \\
\hline 52 C 061 & Ordu-Kabadüz & 406 \\
\hline 52 C 063 & Ordu-Gülyalı & 195 \\
\hline $52 \mathrm{C} 065$ & Ordu-Gülyalı & 357 \\
\hline 52 C 071 & Ordu-Merkez & 524 \\
\hline $52 \mathrm{C} 073$ & Ordu-Perşembe & 229 \\
\hline
\end{tabular}

TABLE 1: Continued.

\begin{tabular}{|c|c|c|}
\hline Accession & Collection area (province-district) & $\begin{array}{c}\text { Altitude } \\
(\mathrm{m})\end{array}$ \\
\hline 52 C 074 & Ordu-Perşembe & 110 \\
\hline $52 \mathrm{C} 078$ & Ordu-Perşembe & 297 \\
\hline 52 C 079 & Ordu-Ünye & 374 \\
\hline 52 C 081 & Ordu-Çaybaşı & 536 \\
\hline $52 \mathrm{C} 087$ & Ordu-İkizce & 341 \\
\hline $52 \mathrm{C} 090$ & Ordu-Akkuş & 1097 \\
\hline 52 C 091 & Ordu-Akkuş & 1198 \\
\hline $52 \mathrm{C} 093$ & Ordu-Akkuş & 965 \\
\hline $52 \mathrm{C} 096$ & Ordu-Fatsa & 331 \\
\hline $52 \mathrm{C} 100$ & Ordu-Ünye & 354 \\
\hline 52 S 001 & Ordu-Kabataş & 450 \\
\hline 52 S 002 & Ordu-Çamaş & 329 \\
\hline $52 \mathrm{~S} 003$ & Ordu-Mesudiye & 1138 \\
\hline 52 S 004 & Ordu-Mesudiye & 1302 \\
\hline $52 \mathrm{~S} 005$ & Ordu-Mesudiye & 1133 \\
\hline $52 S 006$ & Ordu-Perşembe & 0 \\
\hline 52 S 007 & Ordu-Çaybaşı & 430 \\
\hline 52 S 008 & Ordu-Akkuş & 1102 \\
\hline $52 \mathrm{M} 001$ & Ordu-Kabataş & 503 \\
\hline $52 \mathrm{M} 003$ & Ordu-Mesudiye & 1350 \\
\hline $52 \mathrm{M} 005$ & Ordu-Merkez & 376 \\
\hline $52 \mathrm{M} 006$ & Ordu-Akkuş & 1258 \\
\hline $52 \mathrm{M} 007$ & Ordu-Akkuş & 1125 \\
\hline $52 \mathrm{M} 008$ & Ordu-Akkuş & 1223 \\
\hline $52 \mathrm{M} 009$ & Ordu-Akkuş & 1064 \\
\hline 53 C 001 & Rize-İkizdere & 780 \\
\hline $53 \mathrm{C} 002$ & Rize-İkizdere & 968 \\
\hline 53 C 005 & Rize-İkizdere & 838 \\
\hline 53 C 006 & Rize-İkizdere & 701 \\
\hline 53 C 008 & Rize-Güneysu & 518 \\
\hline $53 \mathrm{C} 009$ & Rize-Çayeli & 798 \\
\hline 53 S 001 & Rize-Çamlıhemşin & 1315 \\
\hline $55 \mathrm{C} 002$ & Samsun-Ladik & 958 \\
\hline 55 C 005 & Samsun-Ladik & 754 \\
\hline 55 C 015 & Samsun-Vezirköprü & 749 \\
\hline 55 C 027 & Samsun-Merkez & 722 \\
\hline 55 C 040 & Samsun-Terme & 427 \\
\hline 55 C 049 & Samsun-Asarcık & 1013 \\
\hline 55 C 054 & Samsun-Asarcık & 810 \\
\hline $55 \mathrm{C} 055$ & Samsun-Havza & 1033 \\
\hline 55 C 060 & Samsun-Çarşamba & 658 \\
\hline 55 C 065 & Samsun-Ondokuzmayıs & 247 \\
\hline $55 \mathrm{C} 067$ & Samsun-Bafra & 623 \\
\hline 55 C 072 & Samsun-Alaçam & 683 \\
\hline 55 C 080 & Samsun-Yakakent & 151 \\
\hline 55 C 081 & Samsun-Yakakent & 246 \\
\hline
\end{tabular}


TABLE 1: Continued.

\begin{tabular}{|c|c|c|}
\hline Accession & Collection area (province-district) & $\begin{array}{c}\text { Altitude } \\
(\mathrm{m})\end{array}$ \\
\hline 55 C 083 & Samsun-Terme & 287 \\
\hline 55 C 092 & Samsun-Kavak & 753 \\
\hline 55 C 093 & Samsun-Kavak & 765 \\
\hline 55 C 105 & Samsun-Vezirköprü & 659 \\
\hline 55 C 111 & Samsun-Bafra & 431 \\
\hline 55 C 116 & Samsun-Ayvacik & 691 \\
\hline 55 C 121 & Samsun-Salıpazarı & 1097 \\
\hline 55 C 124 & Samsun-Tekkeköy & 126 \\
\hline 55 C 131 & Samsun-Çarşamba & 328 \\
\hline 55 C 134 & Samsun-Bafra & 510 \\
\hline 55 S 004 & Samsun-Havza & 821 \\
\hline 55 S 008 & Samsun-Ondokuzmayıs & 236 \\
\hline 55 S 011 & Samsun-Yakakent & 685 \\
\hline 55 S 012 & Samsun-Ayvacık & 173 \\
\hline 55 S 015 & Samsun-Çarşamba & 168 \\
\hline $55 \mathrm{~S} 016$ & Samsun-Ladik & 909 \\
\hline 55 S 019 & Samsun-Ayvacık & 630 \\
\hline 55 S 021 & Samsun-Merkez & 560 \\
\hline 55 S 022 & Samsun-Asarcık & 779 \\
\hline 55 M 001 & Samsun-Vezirköprü & 351 \\
\hline $55 \mathrm{M} 003$ & Samsun-Havza & 352 \\
\hline $55 \mathrm{M} 005$ & Samsun-Vezirköprü & 351 \\
\hline $55 \mathrm{M} 006$ & Samsun-Vezirköprü & 332 \\
\hline 55 M 009 & Samsun-Ayvacık & 700 \\
\hline $60 \mathrm{C} 001$ & Tokat-Reşadiye & 1152 \\
\hline $60 \mathrm{C} 005$ & Tokat-Pazar & 1180 \\
\hline $60 \mathrm{M} 001$ & Tokat-Merkez & 655 \\
\hline 60 M 002 & Tokat-Merkez & 673 \\
\hline 60 M 005 & Tokat-Almus & 1074 \\
\hline 60 M 008 & Tokat-Almus & 1004 \\
\hline 60 M 010 & Tokat-Almus & 1004 \\
\hline $60 \mathrm{M} 014$ & Tokat-Almus & 805 \\
\hline 60 M 015 & Tokat-Almus & 796 \\
\hline 60 M 016 & Tokat-Almus & 802 \\
\hline 60 M 017 & Tokat-Almus & 789 \\
\hline 60 M 019 & Tokat-Almus & 817 \\
\hline $60 \mathrm{M} 028$ & Tokat-Niksar & 710 \\
\hline $60 \mathrm{M} 030$ & Tokat-Niksar & 672 \\
\hline 60 M 031 & Tokat-Merkez & 676 \\
\hline $60 \mathrm{M} 033$ & Tokat-Merkez & 834 \\
\hline $60 \mathrm{M} 036$ & Tokat-Pazar & 1013 \\
\hline $60 \mathrm{M} 037$ & Tokat-Pazar & 1044 \\
\hline 60 M 044 & Tokat-Merkez & 783 \\
\hline $61 \mathrm{C} 002$ & Trabzon-Araklı & 215 \\
\hline $61 \mathrm{C} 015$ & Trabzon-Maçka & 992 \\
\hline $61 \mathrm{C} 017$ & Trabzon-Akçaabat & 1714 \\
\hline
\end{tabular}

TABLE 1: Continued.

\begin{tabular}{lcc}
\hline Accession & Collection area (province-district) & $\begin{array}{c}\text { Altitude } \\
(\mathrm{m})\end{array}$ \\
\hline 61 C 020 & Trabzon-Düzköy & 585 \\
61 C 022 & Trabzon-Tonya & 733 \\
61 C 029 & Trabzon-Beşikdüzü & 63 \\
61 S 001 & Trabzon-Araklı & 468 \\
61 S 002 & Trabzon-Merkez & 10 \\
\hline
\end{tabular}

\section{Results and Discussion}

Great genetic diversity was observed among the wild sweet cherry, sour cherry, and mahaleb genotypes collected from Central and East Black Sea Region of Turkey. Several researchers have reported the morphological variation between some Prunus subgenus cerasus genotypes such as for sweet cherry (P. avium), sour cherry (P. cerasus), and mahaleb (P. mahaleb) [4-7].

The morphological traits assessed showed a wide variation. Differences among cherries genotypes based on similarity of morphological characters are shown in Figure 2 using the hierarchical clustering. Unweighted pair group method with arithmetic mean cluster analysis revealed distance indexes between 6.66 and 325.84. A total of 188 genotypes including the selected genotypes and clonal rootstocks were examined for morphological distance. The closest sweet cherry rootstock candidates were $52 \mathrm{C} 071$ and $52 \mathrm{C} 079$ (12.79), while the most distant were 05 C 002 and 08 C 039 (184.29). The closest sour cherries were $53 \mathrm{~S} 001$ and 61S001 (8.75), while the most distant were 08 S002 and $55 \mathrm{~S} 021$ (44.38). The closest mahalebs were $28 \mathrm{M} 001$ and $55 \mathrm{M} 005$ (6.66), while the most distant were $05 \mathrm{M} 001$ and $52 \mathrm{M} 007$ (72.14). The most distant genotypes among species were 05C 002 and 55S012 (325.84). According to the analysis of the morphological index, all of the genotypes were distinguishable from one another. The dendrogram had eight main groups, which had twelve subgroups. The first group consisted of four subgroups and included one hundred seventy five genotypes and Gisela 5, Gisela 6, and Maxma 14 clonal rootstocks. The second group consisted of $08 \mathrm{C} 056$ genotype and PHL-A, Weiroot 158, and Montmorency clonal rootstocks. Other six groups consisted of only one geno-

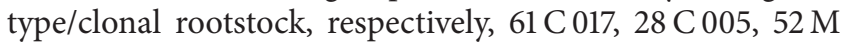
001, 08 C 039, SL 64, and 55 S 012.

Correlations between pomological traits were observed, but these data are not given in the tables in this paper. A characteristic such as plant vigor was positively correlated with leaf blade length (0.38), petiole length (0.34), and petiole thickness (0.36). One-year-old length of internodes was positively correlated with leaf blade length (0.60), petiole length (0.58), and petiole thickness (0.56), while it was negatively correlated with leaf shape $(-0.40)$. One-year-old branching (at the end of summer) was negatively correlated with leaf blade length $(-0.57)$, petiole length $(-0.58)$, petiole thickness $(-0.53)$, and one-year-old length of internodes 
TABLE 2: Morphological characteristics used for the characterization of cherry types.

\begin{tabular}{|c|c|c|}
\hline \multicolumn{3}{|l|}{ Plant } \\
\hline Vigor & PV & Weak (3), medium (5), strong (7) \\
\hline Habit & $\mathrm{PH}$ & Upright (1), spreading (3), drooping (5) \\
\hline Branching & $\mathrm{PB}$ & Weak (3), medium (5), strong (7) \\
\hline \multicolumn{3}{|l|}{ One-year-old shoot } \\
\hline Thickness & ST & Thin (3), medium (5), thick (7) \\
\hline Length & SL & $(\mathrm{cm})$ \\
\hline Length of internodes & SIL & Short (3), medium (5), long (7) \\
\hline First branch height & FBH & $(\mathrm{cm})$ \\
\hline Branch angle & BA & $\left({ }^{\circ}\right)$ \\
\hline Pubescence (upper third) & SP & Absent (1), present (9) \\
\hline Number of lenticels & SLN & Few (3), medium (5), many (7) \\
\hline Anth. coloration of apex & SA & $\begin{array}{c}\text { Absent or very weak (1), weak (3), medium (5), strong (7), very } \\
\text { strong (9) }\end{array}$ \\
\hline Position of vegetative bud & SBP & Adpressed (1), slightly held out (2), markedly held out (3) \\
\hline Size of vegetative bud & SBS & Small (3), medium (5), large (7) \\
\hline Shape of apex of vegetative bud & BAS & Acute (1), obtuse (2), rounded (3) \\
\hline Size of vegetative bud support & BSS & Small (3), medium (5), large (7) \\
\hline Branching (at the end of summer) & SB & Number of branching \\
\hline Intensity of anthocyanin coloration of young leaf & LAI & Weak (3), medium (5), strong (7) \\
\hline \multicolumn{3}{|l|}{ Leaf } \\
\hline Length & LL & $(\mathrm{cm})$ \\
\hline Width & LW & $(\mathrm{cm})$ \\
\hline Ratio length/width & RLW & Very small (1), small (3), medium (5), large (7), very large (9) \\
\hline Shape & LS & Narrow elliptic (1), elliptic (2), circular (3), ovate (4), obovate (5) \\
\hline Angle of apex (excluding tip) & LAA & Acute (1), right-angled (2), obtuse (3) \\
\hline Length of tip & LTL & Short (3), medium (5), long (7) \\
\hline Shape of base & LBS & Acute (1), obtuse (2), truncate (3) \\
\hline Color of upper side & LUC & light green (1), dark green (2), red (3), reddish brown (4) \\
\hline Glossiness of upper side & LUG & Weak (3), medium (5), strong (7) \\
\hline Pubescence of lower side at apex & LLP & Weak (3), medium (5), strong (7) \\
\hline Incisions of margin & LMI & Only crenate (1), both crenate and serrate (2), only serrate (3) \\
\hline Depth of incisions of margin & LMID & Shallow (3), medium (5), deep (7) \\
\hline Petiole length & PL & Short (3), medium (5), long (7) \\
\hline Petiole presence of pubescence & PUP & Absent (1), present (9) \\
\hline Petiole intensity of pubescence & PUPI & Weak (3), medium (5), strong (7) \\
\hline Petiole depth of groove & PGD & Shallow (3), medium (5), deep (7) \\
\hline Petiole thickness & PT & $(\mathrm{cm})$ \\
\hline Ratio length of leaf/petiole & RLPL & small (3), medium (5), large (7) \\
\hline Presence of stipules & LSP & Absent (1), present (9) \\
\hline Stipule length & STL & Short (3), medium (5), long (7) \\
\hline Presence of nectaries & $\mathrm{LN}$ & Absent (1), present (9) \\
\hline Predominant number of nectaries & LNN & One (1), two (2), more than two (3) \\
\hline Position of nectaries & LNP & $\begin{array}{l}\text { predominantly on base of blade (1), equally distributed on base } \\
\text { of blade and petiole (2), predominantly on petiole (3) }\end{array}$ \\
\hline Nectary color & NC & Gren (1), yellow (2), red (3), violet (4) \\
\hline Nectary shape & NS & Round (1), reniform (2) \\
\hline
\end{tabular}


TABLE 3: Eigenvalues and proportions of variance described by the 13 principal components that correspond to eigenvalues greater than 1 .

\begin{tabular}{|c|c|c|c|c|c|c|c|c|c|c|c|c|c|}
\hline & \multicolumn{13}{|c|}{ PC axis } \\
\hline & 1 & 2 & 3 & 4 & 5 & 6 & 7 & 8 & 9 & 10 & 11 & 12 & 13 \\
\hline Eigenvalues & 8,13 & 3,81 & 3,36 & 2,75 & 1,93 & 1,66 & 1,55 & 1,42 & 1,34 & 1,25 & 14 & 1,06 & 1,01 \\
\hline Explained proportion of variation (\%) & 19,35 & 9,08 & 8,00 & 6,54 & 4,60 & 3,96 & 3,68 & 3,37 & 3,19 & 2,98 & 2,71 & 2,52 & 2,41 \\
\hline $\begin{array}{l}\text { Cumulative proportion of variation } \\
(\%)\end{array}$ & 19,35 & 28,43 & 36,43 & 42,97 & 47,56 & 51,52 & 55,20 & 58,57 & 61,76 & 64,74 & 67,46 & 9,98 & 72,38 \\
\hline Characters & \multicolumn{13}{|c|}{ Eigen vectors } \\
\hline Leaf blade: shape & $-0,69$ & 0,33 & 0,10 & 0,10 & 0,17 & $-0,01$ & 0,10 & 0,00 & 0,04 & 0,13 & 0,09 & $-0,10$ & $-0,11$ \\
\hline Leaf blade: angle of apex & $-0,36$ & 0,55 & 0,14 & $-0,22$ & $-0,06$ & $-0,02$ & $-0,07$ & 0,07 & $-0,10$ & $-0,14$ & 0,09 & 0,32 & 0,15 \\
\hline Leaf blade: length of tip & 0,47 & $-0,50$ & $-0,05$ & 0,09 & $-0,08$ & 0,05 & 0,04 & 0,14 & $-0,13$ & 0,03 & 0,05 & $-0,32$ & $-0,11$ \\
\hline Leaf blade: shape of base & $-0,28$ & 0,55 & 0,13 & $-0,15$ & 0,26 & $-0,28$ & 0,03 & $-0,21$ & 0,09 & 0,05 & 0,14 & 0,00 & 0,15 \\
\hline r of upper side & 0,24 & $-0,16$ & $-0,34$ & 0,13 & 0,15 & $-0,30$ & $-0,23$ & 0,39 & 0,19 & $-0,19$ & $-0,02$ & $-0,21$ & 0,24 \\
\hline Leaf blade: $\mathrm{g}$ & $-0,23$ & $-0,12$ & 0,22 & $-0,01$ & 0,12 & 0,22 & 0,47 & $-0,41$ & $-0,12$ & 0,04 & $-0,08$ & $-0,01$ & 0,20 \\
\hline $\begin{array}{l}\text { Leaf blade: pubescence of lower side at } \\
\text { apex }\end{array}$ & 0,36 & 0,19 & $-0,21$ & $-0,04$ & 0,03 & $-0,06$ & 0,16 & 0,24 & 0,05 & 0,37 & 0,34 & 0,04 & 0,33 \\
\hline $\begin{array}{l}\text { Young sh } \\
\text { coloratio }\end{array}$ & 0,49 & $-0,01$ & 0,44 & 0,22 & $-0,06$ & 0,04 & $-0,22$ & $-0,26$ & $-0,18$ & $-0,11$ & 0,35 & $-0,05$ & $-0,09$ \\
\hline Leaf blade: & 0,48 & $-0,10$ & $-0,16$ & 0,09 & 0,10 & 0,15 & 0,13 & $-0,26$ & 0,28 & 0,40 & $-0,12$ & $-0,24$ & $-0,10$ \\
\hline $\begin{array}{l}\text { Leaf blade: depth of incisic } \\
\text { margin }\end{array}$ & 0,42 & $-0,12$ & $-0,13$ & 0,10 & 0,51 & $-0,25$ & $-0,10$ & $-0,04$ & 0,19 & $-0,01$ & $-0,09$ & $-0,01$ & 0,23 \\
\hline Leaf: presence of stipules & $-0,05$ & $-0,21$ & 0,87 & $-0,21$ & $-0,06$ & $-0,03$ & $-0,02$ & 0,21 & 0,05 & 0,12 & $-0,13$ & 0,05 & 0,10 \\
\hline 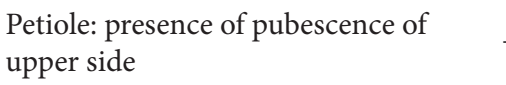 & $-0,03$ & $-0,19$ & 0,87 & $-0,25$ & $-0,05$ & 0,00 & $-0,01$ & 0,19 & 0,06 & 0,15 & $-0,14$ & 0,07 & 0,10 \\
\hline $\begin{array}{l}\text { Petiole: intensity of pubescence of } \\
\text { upper side }\end{array}$ & 0,16 & $-0,34$ & 0,79 & $-0,26$ & 0,10 & 0,00 & $-0,04$ & 0,10 & 0,12 & 0,13 & $-0,14$ & 0,03 & 0,06 \\
\hline th of Groove & 0,54 & $-0,23$ & $-0,06$ & $-0,08$ & 0,41 & $-0,09$ & $-0,04$ & 0,04 & 0,03 & $-0,18$ & $-0,08$ & 0,21 & 0,14 \\
\hline Leaf: pre & 0,09 & 0,18 & 0,27 & $-0,19$ & 0,02 & 0,04 & 0,56 & 0,22 & 0,04 & $-0,32$ & 0,18 & $-0,37$ & $-0,09$ \\
\hline Leaf: predo & 0,53 & 0,30 & 0,13 & $-0,02$ & 0,10 & $-0,15$ & 0,33 & 0,06 & $-0,08$ & $-0,19$ & $-0,05$ & $-0,31$ & 0,17 \\
\hline Leaf: position & 0,26 & 0,43 & $-0,06$ & $-0,20$ & $-0,31$ & 0,09 & 0,01 & 0,37 & $-0,37$ & $-0,01$ & 0,24 & 0,07 & 0,16 \\
\hline Nectary: color & 0,67 & $-0,12$ & 0,03 & $-0,12$ & 0,05 & $-0,06$ & $-0,04$ & 0,13 & $-0,08$ & $-0,07$ & 0,02 & $-0,26$ & $-0,10$ \\
\hline Necta & 0,15 & 0,20 & 0,08 & $-0,08$ & 0,06 & $-0,17$ & 0,30 & 0,10 & 0,42 & $-0,44$ & 0,09 & 0,24 & $-0,25$ \\
\hline Lea & 0,91 & 0,10 & $-0,03$ & 0,08 & 0,06 & $-0,03$ & $-0,02$ & $-0,09$ & $-0,04$ & $-0,08$ & $-0,14$ & 0,14 & $-0,02$ \\
\hline Leaf blade: width & 0,68 & 0,45 & 0,01 & 0,15 & & $-0,08$ & 0,04 & $-0,03$ & 0,03 & $-0,04$ & $-0,16$ & 0,16 & $-0,11$ \\
\hline Leaf blade: ratio le & 0,71 & $-0,42$ & $-0,09$ & $-0,07$ & $-0,10$ & 0,09 & $-0,08$ & $-0,09$ & $-0,11$ & $-0,10$ & $-0,04$ & 0,05 & 0,12 \\
\hline Petiole: 1 & 0,73 & 0,54 & 0,08 & 0,00 & 0,04 & $-0,07$ & $-0,01$ & $-0,14$ & 0,00 & 0,10 & $-0,09$ & 0,02 & $-0,07$ \\
\hline $\begin{array}{l}\text { Leaf: ratio length o } \\
\text { of petiole }\end{array}$ & 0,07 & $-0,76$ & $-0,12$ & 0,12 & 0,01 & 0,10 & $-0,03$ & 0,11 & $-0,05$ & $-0,24$ & 0,00 & 0,11 & 0,08 \\
\hline Petiole: thickness & 0,84 & 0,13 & $-0,01$ & 0,08 & 0,08 & 0,02 & $-0,01$ & $-0,06$ & $-0,02$ & 0,05 & $-0,11$ & 0,10 & $-0,03$ \\
\hline Stipule: le & 0,50 & $-0,19$ & 0,29 & $-0,08$ & 0,03 & $-0,04$ & $-0,07$ & 0,00 & 0,33 & 0,01 & 0,40 & 0,01 & $-0,20$ \\
\hline Plant: vigor & 0,43 & 0,12 & $-0,04$ & 0,02 & 0,10 & 0,51 & 0,18 & $-0,11$ & 0,06 & $-0,10$ & 0,16 & 0,08 & 0,21 \\
\hline Plant: habit & $-0,15$ & 0,15 & $-0,09$ & $-0,04$ & 0,27 & 0,59 & $-0,01$ & 0,15 & 0,32 & 0,07 & $-0,03$ & $-0,04$ & $-0,10$ \\
\hline Plant: branching & $-0,16$ & 0,07 & $-0,05$ & $-0,02$ & 0,37 & 0,64 & $-0,05$ & 0,34 & 0,02 & $-0,01$ & 0,02 & 0,05 & $-0,05$ \\
\hline Pubescence of shoot & $-0,11$ & $-0,08$ & 0,05 & $-0,10$ & 0,61 & $-0,03$ & 0,14 & $-0,10$ & $-0,40$ & $-0,02$ & 0,01 & 0,04 & 0,05 \\
\hline Anthocyanin coloration & 0,53 & $-0,07$ & 0,38 & 0,19 & $-0,07$ & 0,18 & $-0,25$ & $-0,23$ & $-0,13$ & $-0,17$ & 0,33 & 0,01 & $-0,05$ \\
\hline $\begin{array}{l}\text { One-year-old shoot: pos. of veg. bud in } \\
\text { relation to shoot }\end{array}$ & 0,21 & $-0,43$ & $-0,14$ & 0,14 & 0,09 & $-0,12$ & 0,32 & $-0,05$ & $-0,08$ & 0,33 & 0,35 & 0,10 & 0,12 \\
\hline $\begin{array}{l}\text { One-year-old shoot: size of vegetative } \\
\text { bud }\end{array}$ & 0,08 & 0,25 & 0,04 & 0,28 & 0,30 & 0,08 & $-0,06$ & $-0,03$ & 0,34 & $-0,01$ & $-0,09$ & $-0,18$ & 0,42 \\
\hline $\begin{array}{l}\text { One-year-old shoot: shape of apex of } \\
\text { vegetative bud }\end{array}$ & 0,01 & $-0,28$ & 0,05 & 0,25 & 0,24 & 26 & 0,52 & 0,01 & 0,03 & 0,07 & 6 & 0,17 & 0 \\
\hline
\end{tabular}


TABLe 3: Continued.

\begin{tabular}{|c|c|c|c|c|c|c|c|c|c|c|c|c|c|}
\hline & \multicolumn{13}{|c|}{ PC axis } \\
\hline & 1 & 2 & 3 & 4 & 5 & 6 & 7 & 8 & 9 & 10 & 11 & 12 & 13 \\
\hline $\begin{array}{l}\text { One-year-old shoot: size of vegetative } \\
\text { bud support }\end{array}$ & 0,17 & $-0,32$ & $-0,16$ & 0,31 & $-0,10$ & $-0,04$ & 0,20 & 0,25 & 0,19 & 0,15 & 0,14 & 0,38 & $-0,08$ \\
\hline One-year-old shoot: length & $-0,09$ & 0,08 & 0,43 & 0,68 & 0,21 & 0,04 & $-0,04$ & $-0,04$ & 0,06 & $-0,07$ & 0,08 & $-0,01$ & 0,22 \\
\hline One-year-old shoot: thickness & $-0,10$ & 0,27 & 0,24 & 0,75 & 0,05 & $-0,04$ & $-0,18$ & $-0,01$ & 0,21 & 0,04 & 0,08 & $-0,05$ & $-0,02$ \\
\hline $\begin{array}{l}\text { One-year-old shoot: length of } \\
\text { internode }\end{array}$ & 0,70 & 0,27 & $-0,09$ & 0,07 & $-0,09$ & 0,19 & 0,05 & 0,20 & $-0,07$ & 0,18 & $-0,14$ & 0,10 & 0,03 \\
\hline $\begin{array}{l}\text { One-year-old shoot: number of } \\
\text { lenticels }\end{array}$ & 0,12 & 0,11 & $-0,04$ & $-0,55$ & 0,21 & $-0,20$ & $-0,25$ & 0,03 & 0,08 & 0,31 & 0,27 & $-0,09$ & $-0,15$ \\
\hline One-year-old shoot: branching & $-0,66$ & $-0,24$ & 0,04 & 0,39 & 0,32 & $-0,09$ & $-0,06$ & 0,10 & $-0,03$ & $-0,06$ & 0,10 & $-0,04$ & 0,05 \\
\hline First branch height & 0,56 & 0,32 & 0,18 & 0,27 & 0,01 & $-0,02$ & 0,02 & 0,22 & $-0,21$ & 0,16 & $-0,19$ & 0,00 & $-0,18$ \\
\hline Branch angle & $-0,20$ & 0,14 & 0,17 & 0,61 & 0,20 & $-0,14$ & 0,04 & 0,30 & $-0,31$ & 0,13 & $-0,07$ & $-0,04$ & $-0,16$ \\
\hline
\end{tabular}

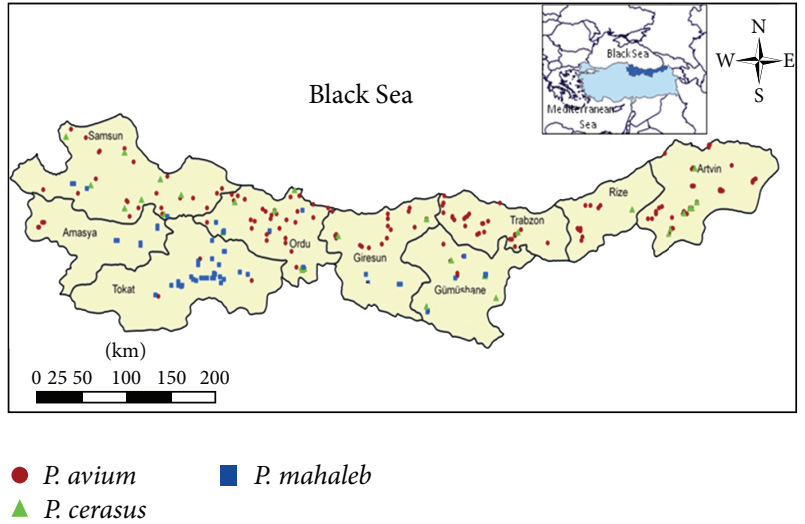

FIGURE 1: Map of the distribution for genotypes in Central and East Black Sea Regions in Turkey.

$(-0.51)$. Information regarding the associations and correlations between different plant characteristics are valuable for breeding programs.

Principal component analysis (PCA) was used to examine the variation of cherries genotypes/clonal rootstocks. Morphological characterization is necessary for the description and classification of germplasm and statistical methods like principal components analysis are useful tools for screening the accessions of a collection $[8,9]$. It allows for visualization of the differences among the individuals, identification of possible groups, and relationships among individuals and variables [10]. The first thirteen axes accounted for $72.38 \%$ of the variability among 188 accessions (Table 3 ). The first PC axis accounted for $8.13 \%$ of the variation, whereas the second, third, and fourth axes accounted for $3.81 \%, 3.36 \%$, and $2.75 \%$, respectively. The first axis was mainly related to leaf blade length (0.91), petiole: thickness $(0.84)$, petiole length $(0.73)$, leaf blade: ratio length/width (0.71), and one-year-old shoot: length of internodes (0.70). The second axis was concerned with leaf: ratio length of leaf blade/length of petiole $(-0.76)$. The third axis was mainly concerned with leaf: presence of

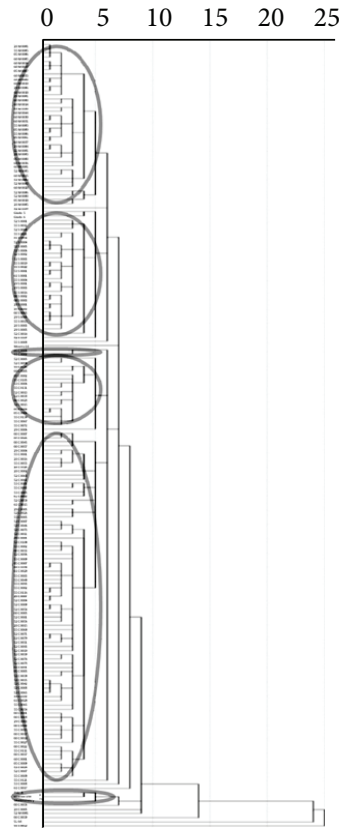

FIGURE 2: Cluster analysis for 188 genotypes/clonal rootstocks based on morphological data.

stipules (0.87), petiole: presence of pubescence of upper side (0.87), and petiole: intensity of pubescence of upper side (0.79). The remaining ten axes were related to other leaf, shoot, and plant traits (Table 3 ).

The populations were grouped into seventeen clusters by cluster analysis. These are composed of six groups and eleven single genotypes. The different cherry genotypes identified based on the similarity of their morphological characteristics and their hierarchical clustering are shown in Figure 2. These seventeen groups can be considered as distinct germplasm pools (Figure 2). According to diversity analysis of coefficients, the $28 \mathrm{M} 0001$ and $55 \mathrm{M} 0005$ genotypes were found to be very similar (6.66), while the $05 \mathrm{C} 0002$ and $55 \mathrm{~S} 0012$ genotypes were determined as the most distant 


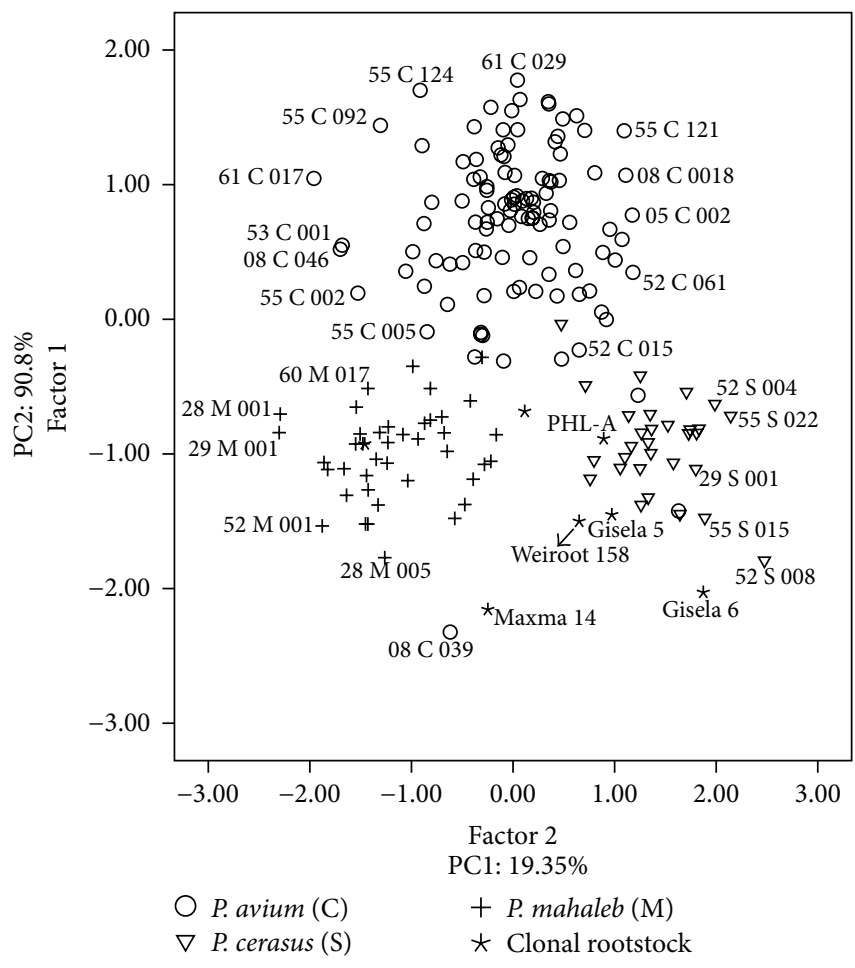

(a)

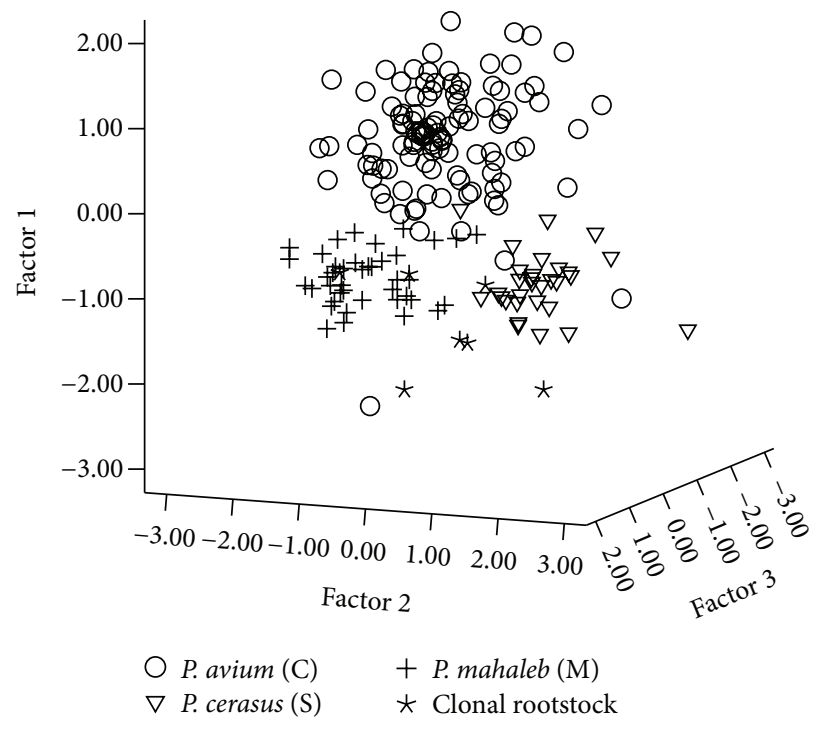

(b)

FIGURE 3: 2D and 3D scatter diagrams of the relationships between the genotypes/clonal rootstocks (based on morphological traits).

genotypes (325.84) in terms of morphological variability. Shahi-Gharahlar et al. [11] reported that dendrogram obtained from morphological traits clearly distinguished the Cerasus subgenus genotypes from the other genotypes. PérezSánchez et al. [4] suggested that a dendrogram obtained from morphological characteristics clearly showed the relationships between cultivars of sweet, sour, and duke cherries.

The high total variance explained by the first three axes was shown in a 2D and 3D screen plot; each genotype/clonal rootstock was plotted based on its principal components score (the cumulative proportion of variance) for each of the first three axes (Figure 3).

\section{Conclusions}

As a result, it can be said that the studied genotypes are diverse and display great variations. The collection, evaluation, and characterization of Turkish cherries germplasm are a field of interest and are of economical and ecological importance. This provides rootstocks with good adaptations to diverse climatic and soil conditions of Turkey. The results may serve as a significant reference for the comparison of genetic resources, the characterization of cherry genotypes, and the cherry rootstock breeding programs to select the best parents with the highest variation. In conclusion, the genotypes evaluated in this study may be useful for both breeders and rootstock breeding programs.

\section{Acknowledgment}

The authors are grateful to the Scientific and Technological Research Council of Turkey (Project no.: TUBITTAK 106O031) for the financial support.

\section{References}

[1] Food and Agricultural Organization (FAO), February 2013, http://faostat.fao.org/site/567/DesktopDefault.aspx?PageID= 567.

[2] S. Ercisli, A. Esitken, E. Orhan, and O. Ozdemir, "Rootstocks used for temperate fruit trees in Turkey: an overview," Sodininkyste ir Darzininkyste, vol. 25, pp. 27-33, 2006.

[3] E. Cromwell, "Agricultural, biodiversity and livelihood: issue and entry point: final report," April 1999, http://www.odi.org .uk/sites/odi.org.uk/files/odi-assets/publications-opinion-files/ 8286.pdf.

[4] R. Pérez-Sánchez, M. A. Gómez-Sánchez, and R. MoralesCorts, "Agromorphological characterization of traditional Spanish sweet cherry (Prunus avium L.), sour cherry (Prunus cerasus L.) and duke cherry (Prunus $\times$ gondouinii Rehd.) cultivars," Spanish Journal of Agricultural Research, vol. 6, no. 1, pp. 42-55, 2008.

[5] A. Khadivi-Khub, Z. Zamani, and N. Bouzari, "Evaluation of genetic diversity in some Iranian and foreign sweet cherry cultivars by using RAPD molecular markers and morphological traits," Horticulture Environment and Biotechnology, vol. 49, no. 3, pp. 188-196, 2008. 
[6] E. G. Moghadam and A. Khalighi, "Relationship between vigor of Iranian Prunus mahaleb L. selected dwarf rootstocks and some morphological characters," Scientia Horticulturae, vol. 111, no. 3, pp. 209-212, 2007.

[7] V. Rakonjac, M. F. Akšić, D. Nikolić, D. Milatović, and S. Čolić, "Morphological characterization of "Oblačinska" sour cherry by multivariate analysis," Scientia Horticulturae, vol. 125, no. 4, pp. 679-684, 2010.

[8] C. Cantini, A. Cimato, and G. Sani, "Morphological evaluation of olive germplasm present in Tuscany region," Euphytica, vol. 109, no. 3, pp. 173-181, 1999.

[9] M. L. Badenes, J. Martínez-Calvo, and G. Llácer, "Analysis of a germplasm collection of loquat (Eriobotrya japonica Lindl.)," Euphytica, vol. 114, no. 3, pp. 187-194, 2000.

[10] J. Martínez-Calvo, A. D. Gisbert, M. C. Alamar et al., "Study of a germplasm collection of loquat (Eriobotrya japonica Lindl.) by multivariate analysis," Genetic Resources and Crop Evolution, vol. 55, no. 5, pp. 695-703, 2008.

[11] A. Shahi-Gharahlar, Z. Zamani, M. R. Fatahi, and N. Bouzari, "Assessment of morphological variation between some Iranian wild Cerasus sub-genus genotypes," Horticulture Environment and Biotechnology, vol. 51, pp. 308-318, 2010. 

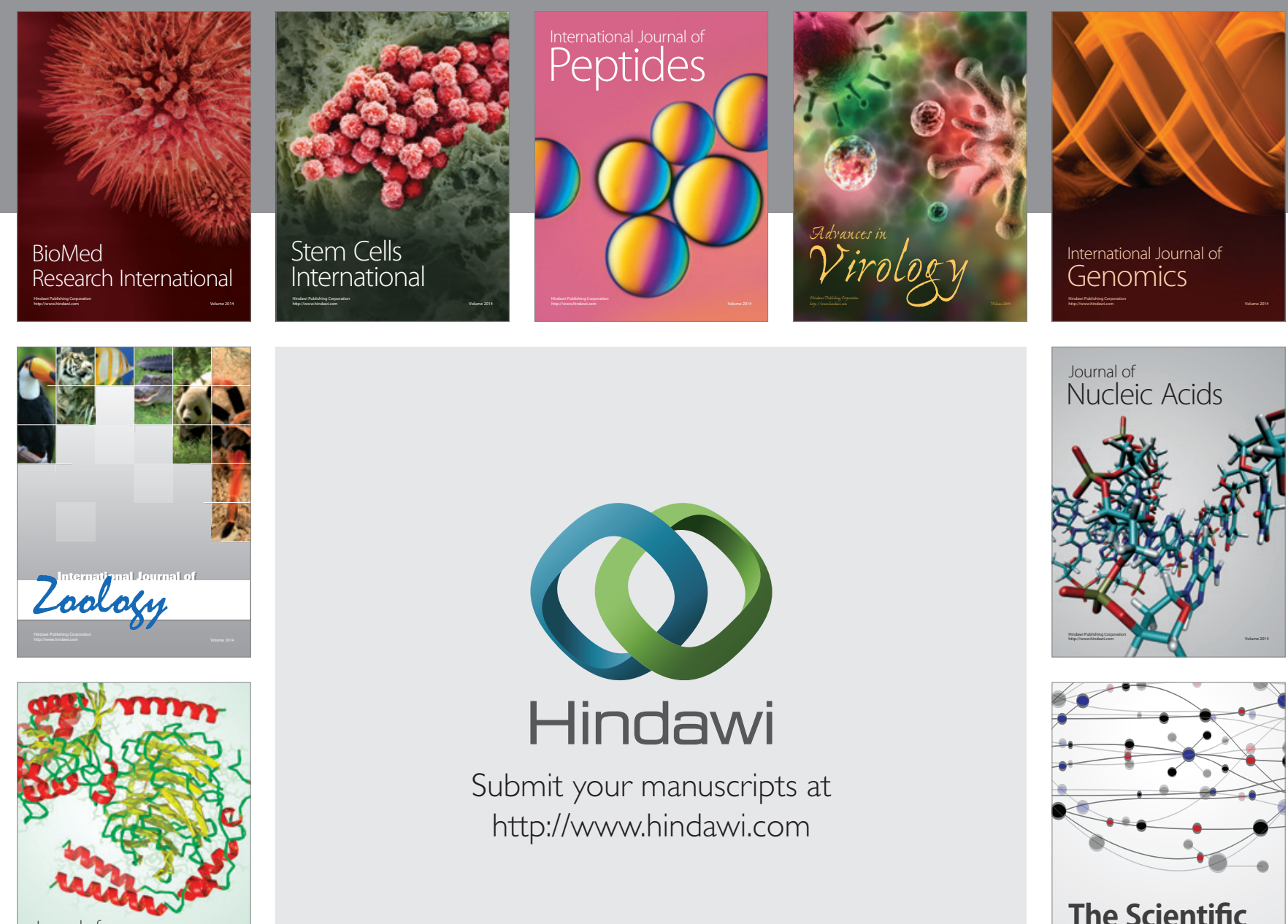

Submit your manuscripts at

http://www.hindawi.com

Journal of
Signal Transduction
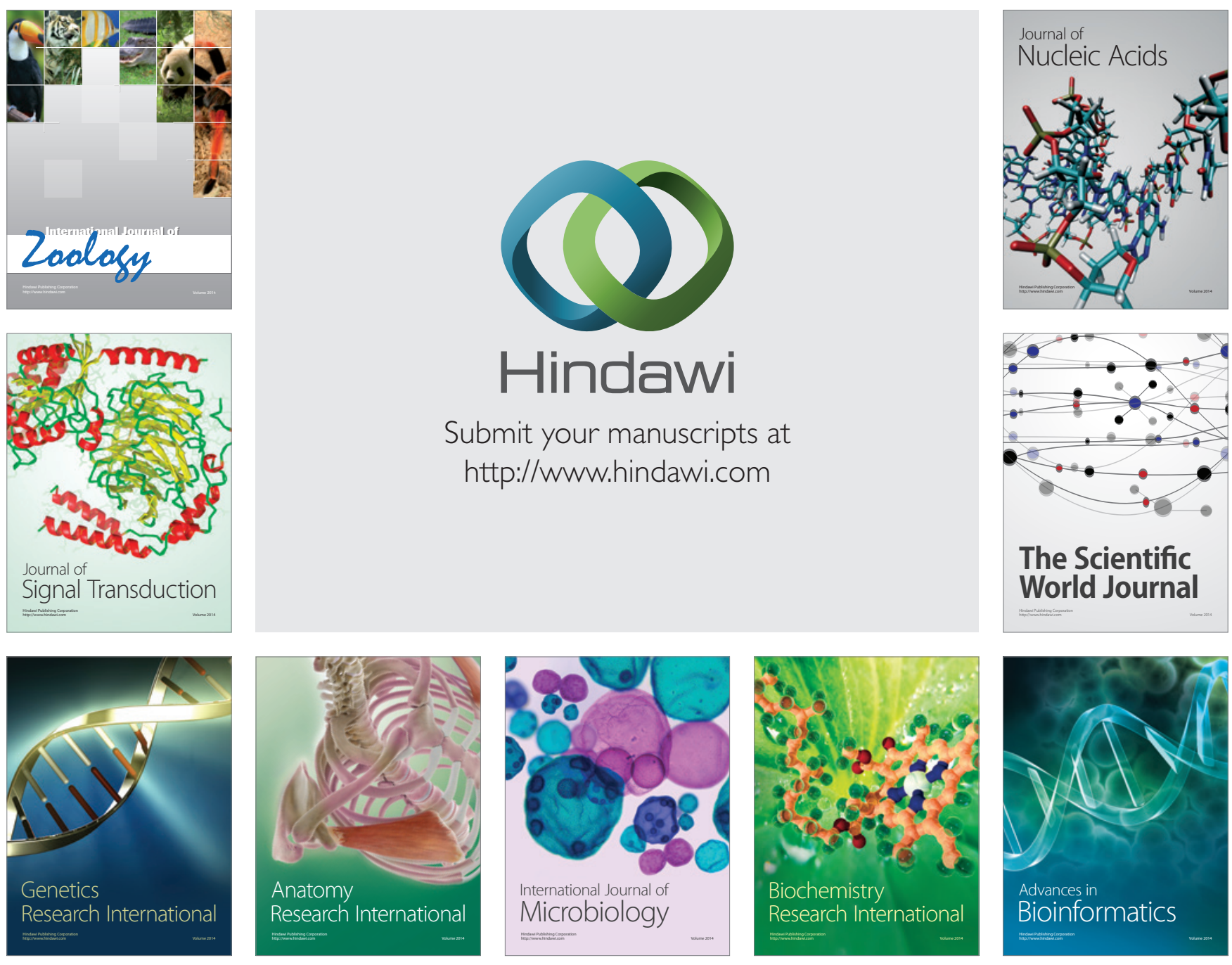

The Scientific World Journal
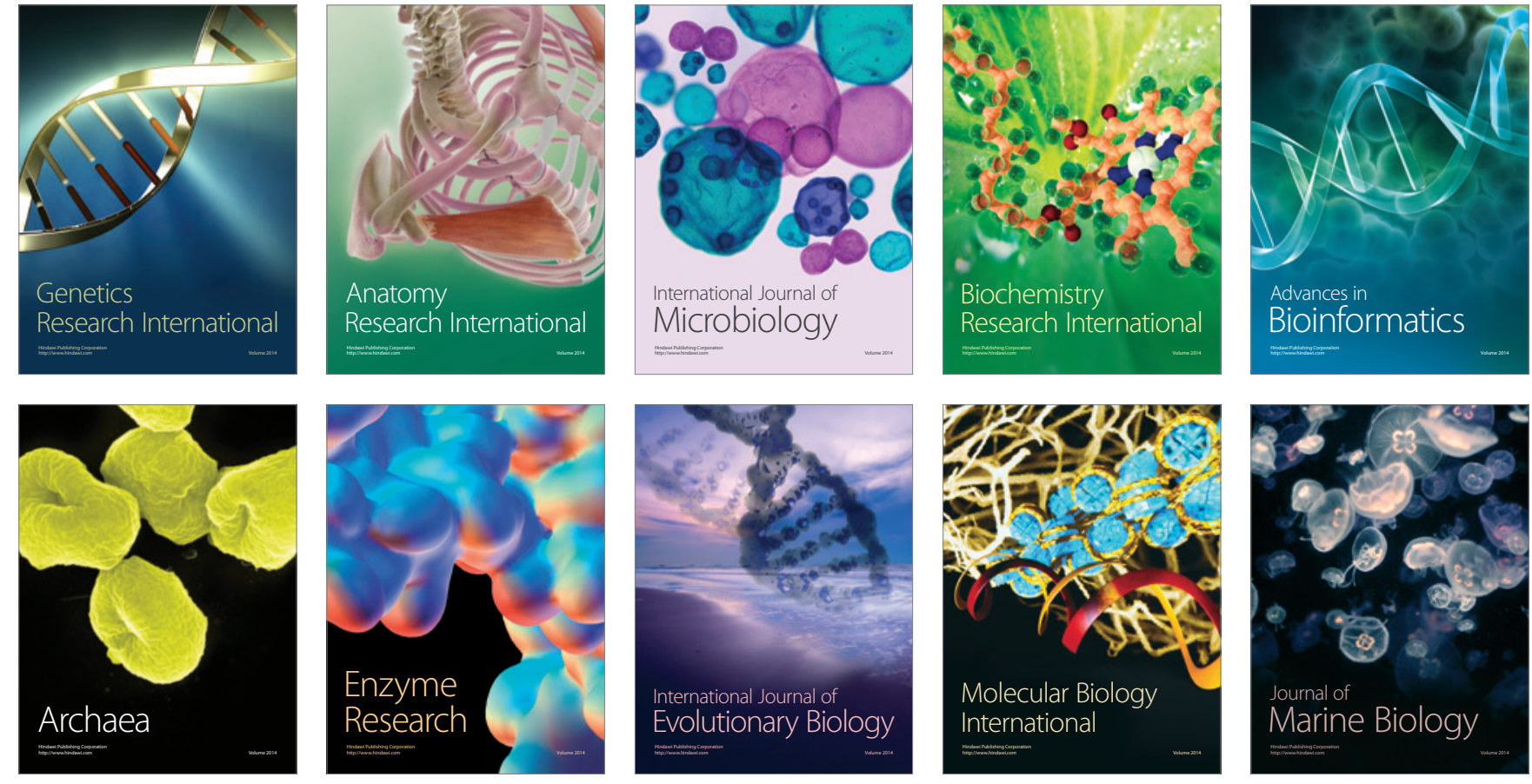